\title{
Bovine Neurobrucellosis: Pathological and Bacteriological Studies
}

\author{
Kh. A. El-Nesr ${ }^{1}$, E. A. Mahdi ${ }^{1}$, W. H. Hamdi ${ }^{2}$, A. H. Abed ${ }^{2}$ \\ ${ }^{1}$ Department of Pathology and ${ }^{2}$ Department of Microbiology, Faculty of Veterinary Medicine, \\ Beni-Suef University, Beni-Suef 62511, Egypt
}

\begin{abstract}
Thirty two cattle, had no neurological syndrome, were serologically positive to brucellosis by using Tube agglutination and Rose Bengal Tests, by the official veterinary authority in Beni Suef province, Egypt. These animals were slaughtered in Beni Suef abattoirs during the project of control and eradication of brucella positive animals in Beni Suef province. Postmortem examination was performed and the brain was mechanically removed. Longitudinally the brain was cut; one half was fixed in formalin $25 \%$ for 2 weeks and the other one were sent to microbiology department for bacteriological isolation. Transverse sections were done in the fixed tissue and samples were collected from cerebrum, cerebellum, medulla oblongata, thalamus, hypothalamus, and caudate nucleus. These samples were processed according to Bancroft and Gamble (2008). From the thirty two brain samples, no isolates of brucella species were recovered. From the thirty two brain samples, no isolates of brucella species were recovered. Brains of slaughtered animals showed no pathological lesions grossly. Microscopically, inflammatory reactions, degeneration, malacia, demyelination, pigmentation, and vascular changes were detected.
\end{abstract}

Brucellosis is one of the most important zoonotic diseases worldwide, particularly in developing countries (Trujillo et al., 1994), Mediterranean countries (Godfroid and Käsbohrer, 2002) and Central Asia (Pappas et al., 2006). It affects animals as the primary host and humans as the secondary host (Al-Kawi, 1995)

Bovine brucellosis is an economically important bacterial disease caused by Brucella abortus, facultative intracellular gram-negative coccobacilli, less frequently Brucella melitensis, and rearly Brucella Suis. In Egypt Brucella melitensis is incriminated to be the causative agent of human and animal brucellosis (Afifi et al., 2005). Infection occurs most commonly via the digestive tract, the usual source of the organism being an infected placenta or aborted fetus. After ingestion, the bacteria are internalized by the M-cells in the Peyer's patches; they then spread to the regional lymph nodes where, as facultative intracellular microorganisms, they proliferate within macrophages (Ackermann et al., 1988). Subsequently, they spread via the bloodstream to other tissues. They localize in certain organs especially the placenta, mammary glands, supramammary in cows and in the testis epididymes in bulls (Jones and Hunts, 1998). Previously, the reticuloendothelial system was the target to brucellosis (Seidel et al., 2003).
Recently, different types of granulomatous reactions were recorded in liver, kidneys and lungs of cattle infected with brucellosis including tuberculous like granuloma, epithelioid cell and giant cell reactions (El-Nesr, 2006).

Central nervous system (CNS) involvement is a serious complication of brucellosis and the clinical presentation is quite heterogeneous (Akdeniz et al., 1998). Although neurobrucellosis is rare, its still a problem throughout the world; particularly in the Mediterranean Basin. Various discrepancies in the diagnosis and management of the disease still exist. The data and the literature are generally restricted to case reports and series.

Neurobrucellosis occurs in $5-10 \%$ of cases of brucellosis and affects the central or peripheral nervous system (Tuncel et al., 2008). This may lead to a variety of clinical manifestations and imaging abnormalities that mimic other neurologic diseases (McLean et al., 1992). These manifestations are diffuse encephalopathy or /meningoencephalitis ,inflammatory peripheral neuritis/radiculitis, inflammatory demyelinative syndromes, papilledema or papillitis without other focal features, meningomyelitis, posterior fossa (ataxic or brainstem) syndromes, and neuropsychiatric syndromes (Banerjee et al., 1999).

Laboratory detection and identification of Brucella species is based largely on culture 
isolation and phenotypic characterization (Probert et al., 2004).

In publications, involvement of nervous tissue in cattle was rarely recorded in contrast to human. The aims of present work were to study the neuropathological changes in the brain of cattle serologically positive to brucellosis and also to isolate the causative agent.

\section{Material and methods}

Animals. Thirty two cattle, had no neurological syndrome, were serologically positive to brucellosis by using Tube agglutination and Rose Bengal Tests, by the official veterinary authority in Beni Suef province, Egypt. These animals were slaughtered in Beni-Suef abattoirs during the project of control and eradication of brucella positive animals in Beni-Suef province. Postmortem examination was performed and the brain was mechanically removed. Longitudinally the brain was cut; one half was fixed in formalin $25 \%$ for 2 weeks and the other one were sent to microbiology department for bacteriological isolation. Transverse sections were done in the fixed tissue and samples were collected from cerebrum, cerebellum, medulla oblongata, thalamus, hypothalamus, and cudate nucleus. These samples were processed according to Bancroft and Gamble (2008).

Histopathological study. Sections of 4-6 microns were stained with Heamtoxylin and Eosin according to Bancroft and Gamble, (2008).

Bacterial isolation. Brain samples (including meninges and parenchyma combined) collected aseptically from cows were examined after storage at $-20^{\circ} \mathrm{C}$. Thawed tissues were macerated in a stomacher (Seward Medical, Worthing, UK), then cultured on 7\% blood agar (Oxoid, $\mathrm{CM}$ 271) and Brucella Selective Medium (Brucella Medium Base [Oxoid, CM 169] supplemented with Brucella Selective Supplement (Oxoid, SR83) and incubated at $37^{\circ} \mathrm{C}$ in an atmosphere containing $\mathrm{CO}_{2} 8 \%$ for 12 days and examined daily for the presence of colonies (Xavier et al., 2009).

\section{Results}

Bacterial isolation. From the thirty two brain samples, no isolates of brucella species were recovered.

Pathological alterations of the brain. Brains of slaughtered animals showed no pathological lesions grossly. Microscopically, inflammatory reactions, degeneration, malacia, demyelination, pigmentation, and vascular changes were detected. Various pathological changes of the brain and numbers of animal affected are shown in (Table 1).

Table (1): Various pathological changes of the brain.

\begin{tabular}{lc}
\hline Lesions & Number \\
\hline Meningitis & 1 \\
Degeneration, malacia and demyelination & 3 \\
Pigmentation & 4 \\
Vascular changes & 6 \\
Encephalitis & 1 \\
\hline
\end{tabular}

Changes in the meninges. Grossly, no changes except congestion of the meningeal blood vessels were found. Microscopically, moderate lymphocytic meningitis was found in only one case and it was characterized by infiltration of moderate numbers of mononuclear cells (Fig. 1). Blood vessels distributed in the pia-arachnoid became dilated and engorged with blood. At many places, the arachnoid was widely separated from the underlying pia matter and the space was partially filled with inflammatory exudate. The mesothelial lining of the pia matter appeared swollen and enlarged. The leptomeninges of cerebral parenchyma had a large number of inflammatory cells consisting of macrophages, and lymphocytes. Vasculitis was observed this case that had meningitis, the tunica adventitia of meningeal blood vessels showed adventitial proliferation with mononuclear cell infiltration.

\section{Pathological changes of brain substance:} a- Neuronal degeneration. No gross pathological changes could be seen in the in cases that had neuronal degeneration. Microscopically, in the cerebral gray matter in the cortical laminae and aggregates of neurons in the basal ganglia (caudate nucleus, putamen, globus pallidus, and subthalamic nucleus). The cytoplasm of neuronal cell body was shrunken, deeply eosinophilic, and frequently angular to triangular in shape. The nucleus was reduced in size, often triangular and pyknotic. The nucleolus and Nissil's substances were not detectable. In H\&E staining sections, the affected somata were pink or red in color. Dead neurons were surrounded by neuronphagia (Fig.2). The degenerative changes varied from multifocal to diffuse.

b- Encephalomalacia. Microscopical examination of this brain revealed areas of parenchymal necrosis. Neurons, glial cells, axons and myelin were necrotic and such foci quickly liquefied (Fig.3). Capillaries were prominent and surrounded by moderate to severe dilatation of the Virchow-Robin space. Demyelination of nerve fibers in the white matter was 
demonstrated by special staining using Kluver Barrera luxol fast blue method. Demyelination was evident in the white matter of both cerebrum and cerebellum and also in the midbrain areas. Phagocytosis and removing of debris proceeded,

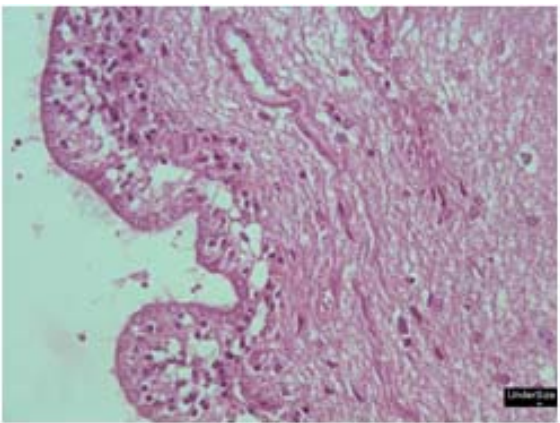

jpg.1

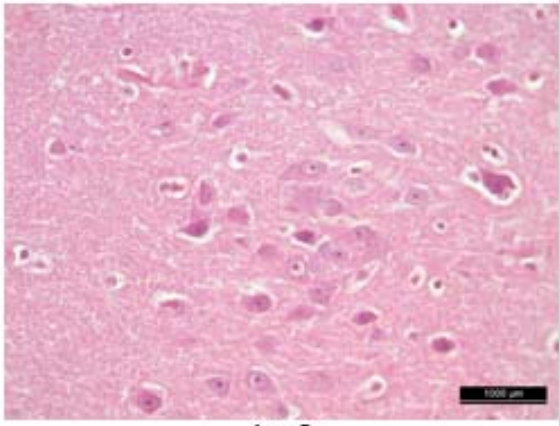

jpg.3

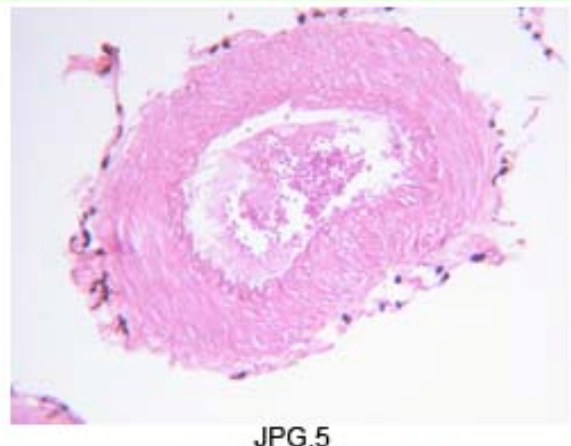

JPG.5

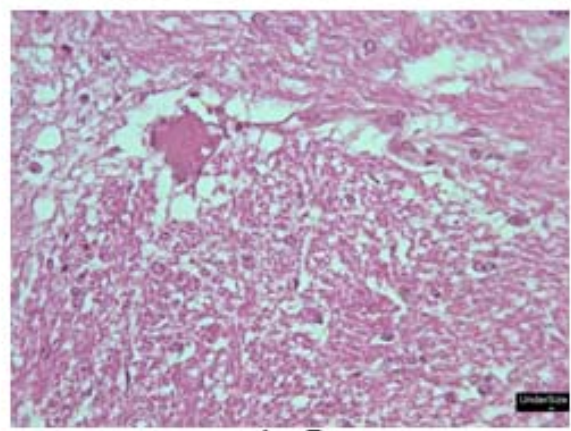

jpg.7 leaving loose cystic cavity populated with gitter cells and capillaries. In the adjacent, less injured tissue astrogliosis was found with few mononuclear cells.

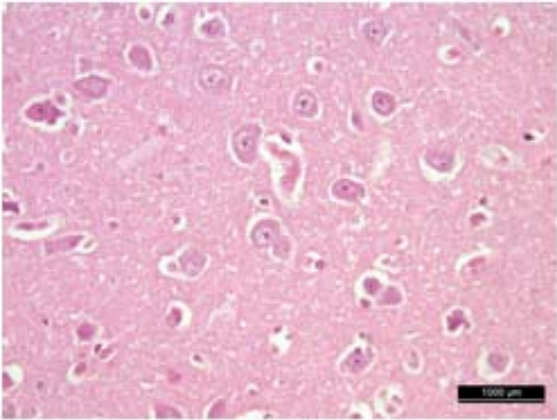

jpg.2

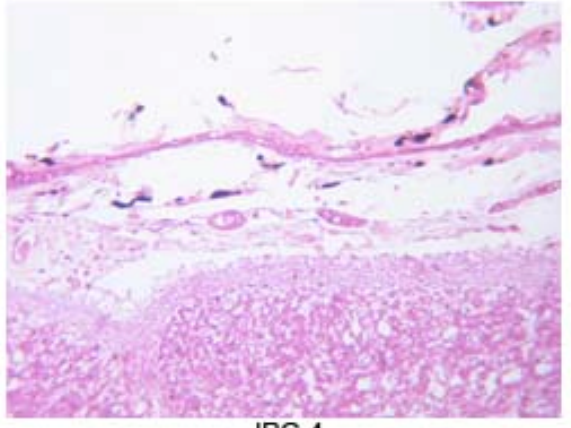

JPG.4

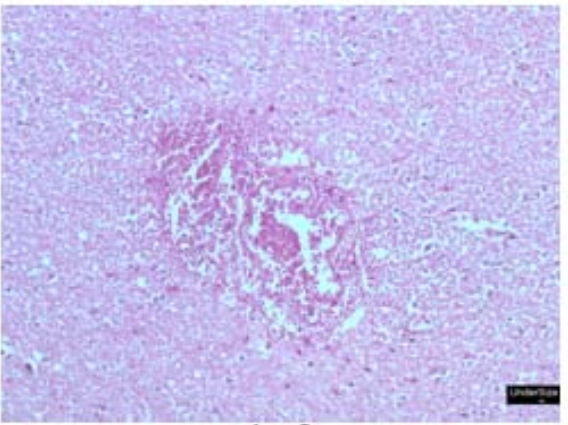

jpg.6

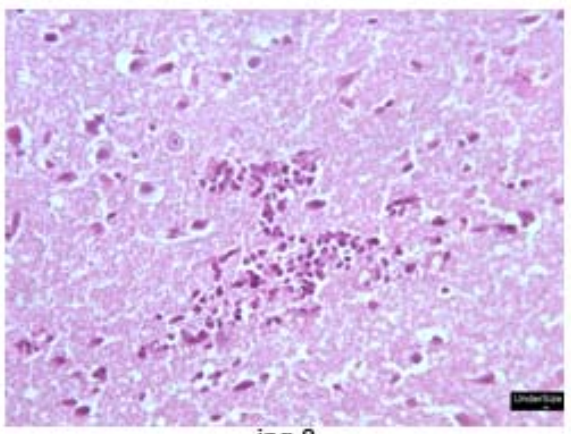

jpg.8

Fig. (1): Pia matter is infilterated with moderate numbers of mononuclear cells (H\&E-X400).

Fig. (2): Cerebral grey matter is showing neuronphagia (H\&E-X400).

Fig. (3): Liquefaction and necrosis of the nervous tissue (H\&E- X400).

Fig. (4): Neuromelanin pigment is present within pia matter (H\&E-X100).

Fig. (5): Neuromelanin pigment is present within the adventitia of a large meningeal blood vessel (H\&E-X400).

Fig.(6): Cerebral grey matter is showing congestion and hemorrhage (H\&E X400).

Fig. (7): Cerebral grey matter is showing perivascular and pericellular edema(H\&E-X400).

Fig. (8): Cerebral grey matter is showing increased number of mononuclear cells; a mixture of glial cells (gliosis), lymphocytes and macrophage (H\&E X400). 
c- Parenchymal and meningeal pigmentation. Neuromelanin accumulated as fine brownish granules in the cytoplasm of certain neurons of the midbrain was commonly seen in 4 cases. This pigment was also present within pia matter and within the adventia of its blood vessels (Fig. $4,5)$.

d- Vascular changes. The most common vascular changes observed in many cases were congestion, hemorrhage, and edema. Some of these changes were associated with other pathological abnormalities as inflammation and degeneration.

Congestion of the brain was manifested by dilatation of the cerebral blood vessels that was seen on expensing the brain tissue. Microscopically congestion was observed in different areas of the brain in most cases throughout the present study including the degree of hemorrhage varied widely. It was confined only to meninges in some cases, whereas it was very extensive involving all the different layers of the cerebrum, cerebellum and midbrain (Fig.6).

The gross appearance of cerebral edema depended on its cause, severity and duration. Moderate to severe edema led to brain swelling which appeared as flattening of the cerebral cortical gyri and slight narrowing of sulci.

Microscopically, edema appeared as several areas of spongy appearance distributed throughout the cerebral cortex .Edema was seen both in white and gray matter of the cerebrum, cerebellum and midbrain; the white matter was more severely affected. In stained sections, it was exhibited as wide inter-fascicular spaces in the white matter. Edema was probably developed to some degree in all pathological states, whether degenerativeor inflammatory. In case of perivascular edema, the perivascular spaces were widened as a result of fluid leakage through the blood vessels (Fig.7) A similar change was seen around the neurons in case of pericellular edema.

\section{e- Inflammatory changes of the brain.}

Non-Suppurative lymphocytic encephalitis. There was no gross picture except mild cerebral congestion and hemorrhage. Microscopically, in the affected brain, mononuclear cells accumulated peripherally within and surrounding the vascular walls in the Virchow-Robin space (perivascular cuffing) was observed, mainly by lymphocytes, in the brain tissue and an infiltration of similar inflammatory cells in the cerebellar meninges. There was also an increased cellularity of the parenchyma caused by an increased number of mononuclear cells, likely a mixture of glial cells (gliosis) mainly lymphocytes and macrophages (Fig.8). Many of these cells have a greatly elongated, often tortuous nucleus probably represent reactive microglial cells, and presumably also macrophages from the peripheral circulation. Neuronal degeneration and necrosis, associated with satelletosis, neuronphagia occurred.

\section{Discussion}

Nervous system involvement in brucellosis might be due to the persisting intracellular microorganism, or perhaps the infection triggers an immune mechanism leading to neuropathology (Akdeniz et al., 1998). In fact, the detection of neurobrucellosis is based on the existence of a neurological picture not explained by any other neurological disease, evidenced by systemic brucellar infection, and the presence of inflammatory alteration in the CSF. Although positive culture is the gold standard for diagnosis, it has often been thought to be suboptimal (Zaidan and Al-Tahan, 1999; Shaalan et al., 2002).

Neurobrucellosis is a neurological complication, first reported by Hughes in 1897 (according to Sanchez-Sousa et al., 1990), which is directly attributable to the presence of Brucella or its products in the central nervous system. This neurological complication can arise at different moments in the evolution of brucellosis. In most cases diagnosis is made between two and 12 months after onset of symptoms. The reported incidence of neurobrucellosis varies from $0.5 \%$ to $25 \%$ of all brucellosis (Colmenero et al., 1983). This large variation is attributable to the lack of standardization of diagnostic criteria used by different authors. Meningitis is the most clinical presentation of neurobrucellosis and meningovascular complications (Bingöl and Togay-Işıay, 2006). According to standard criteria (Labrisseau et al., 1978) meningeal brucellosis was considered when at least one of the following criteria was present: isolation of Brucella from cerebrospinal fluid; presence in cerebrospinal fluid of antiBrucella antibodies at any titre obtained with the microagglutination, Coombs' or Rose Bengal tests; and abnormalities in cerebrospinal fluid (Sanchez-Sousa et al., 1990).

In human, involvement of CNS is seen in $4 \%$ to $13 \%$ of patients with brucellosis (Tuncel et al., 2008; Kochan et al., 2000; puscual et al., 1988Lulu et al., 1988). The manifestation of neurobrucellosis in human is encephalopathy, 
minigioencephalitis, neuritis (Tuncel et al., 2008) and leukoencephalopathy (Seidel et al., 2003). In Egypt brucella meningitis was firstly reported by Mansour et al., (2009) who isolated brucella melitensis and confirmed his results by ELISA and PCR.

Generally, animals infected with brucellosis rarely manifest clinical signs however they were serologically positive, so the infection may pass unnoticeable in non pregnant animals (Calvoet et al., 2007). Abortion, orchitis and epididymitis are the most common features of brucellosis in animals. In contrast to human, severe illness with obvious clinical signs was noticed (Pappas et al., 2005). Nervous manifestations are considered highly reliable indicators for the presence of brain lesions. However some of the brain lesions may not manifest nervous signs. Our cases had no neurological sings.

The nervous system is composed of two types of tissue. Both of which are involved in varying degree in disease processes (nerve cells and neurological cells, and meninges, blood vessels and phagocytic cells.

Several studies reported neuropathological changes in cattle, sheep and pigs in the form of vacuolations, spheroids and diffuse inflammatory changes (Kikuchi et al., 1928; Jeffrey,1992; Hooper,1999; Gavier et al., 2001) but no records have been published describing the neuropathological alterations associated with brucellosis in cattle. The most common histopatholgical changes demonstrated in the nervous system of dolphins infected with brucellosis were meningioencephalitis, and nonsuppurative meningitis. The predominant cellular infiltrates were lymphocytes, plasma cells and macrophages (Hernandez-Mora et al., 2008). No evidence of vasculitis or granulomatous inflammation was detected (Seidel et al., 2003).

In brain tissue, granuloamtous to pyogranulomatous reactions could be observed in mycotic infection and some bacterial infections as Mycoplasma bovis and also in case of migrating helminthes (Jubb et al., 2007).

The laboratory diagnosis of neurobrucellosis is problematic, since Brucellae are rarely isolated from human cerebrospinal fluid (Cooke and Beeching, 2008). In animals, Brucella species were isolated from the brain of dolphins with meningoencephalitis and detected brucella antigen with the immunohistochemical (Gonzàlez et al., 2002).

Concerning bovine (or even ovines) brucellosis, isolation of Brucella species from animal brain was not reported till now. There are very few reports on lesions in the brain in naturally occurring B. abortus infection in bovine foetuses (Guarda, 1977; Hong et al., 1991). This paper reports a case of granulomatous meningitis in the brain of an aborted bovine foetus naturally infected with $\mathrm{B}$. abortus. Çiten et al., (2004) isolated of B. abortus biotype 1 in pure culture from the brain and other samples of aborted bovine foetus. On the contrary, Sözmen et al., (2004) did not isolate B. abortus from the bran but they recovered B. abortus from abomasal contents and lung of aborted bovine foetus.

\section{References}

Ackermann, M. R.; Cheville, N. F. and Deyeoe, B. L. (1988): Bovine ileal dome lymphoepithelial cell: endocytosis and transport of Brucella abortus strain 19. Vet Pathol, 25: 28-35.

Akdeniz, H.; Irmak, H.; Anlar, O. and Demiroz, A. P. (1998): Central nervous system brucellosis: presentation, diagnosis and treatment. J Infect, 36:297-301.

Al-Kawi, M. Z. (1995): Brucellosis. In: Moher JP, Gautier J, eds. Guide to Clinical Neurology, Churchill Livingstone; New York: 677-680.

Banerjee, T. K.; Pal, A. K. and Das, S. (1999): Neurobrucellosis presenting as acute meningoencephalitis. Neurol Ind., 47:160.

Bingöl, A. and Togay-Işıkay, C. (2006): Neurobrucellosis as an exceptional cause of transient ischemic attacks. Eur. J. Neurol., 13:544-548

Çiten, C.; Cangül, I.T.; Akan, M.; Özel, E. and Akkoç, A. (2004): Meningitis associated with Brucella abortus in an aborted bovine foetus. Turk. J. Vet. Anim. Sci., 28: 451453.

Colmenero, J. de D.; Valdivieso, P.; Camps, M. T.; Porras, J. J.; Trujillo, J. and Juarez, C. (1983): Brucelosis: estudio clidemiologico de 139 casos. Med Clin, 80: 748-751.

Cooke, R. P. D. and Beeching, N. J. (2008): Neurobrucellosis in the UK. Brit. Infect. Soc. Published by Elsevier Ltd.

Deniz, T.; Hasan, U.; Mustafa, G. and Uygar, U. (2008): Neurobrucellosis, Eur J Gen Med, 5(4): 245-248

Gavier-Widen, D; Wells, G. A. H.; Simmons, M. M.; Wilesmith, J. W. W. and Ryan, J. (2001): Histological observations on the brains of symptomless 7-year-old cattle. J Comp Pathol, 124:52-59.

El-Nesr, Kh.; Mahdy, E. A.; Shalaby, A. A. and Deeb, S. (2007): Pathological and immunohistochemical studies brucella meletensis in cows. Vet Med J , Giza 55(1): 275292

Godfroid, J. and Käsbohrer, A. (2002): Brucellosis in the European Union and Norway at the turn of the twenty-first century. Vet Microbiol 90: 135-145.

Gonzàlez, L.; Patterson, I. A.; Reid, R. J.; Foster, G.; Barberán, M.; Blasco, J. M.; Kennedy, S.; Howie, F. E.; Godfroid, J.; MacMillan, A. P.; Schock, A. and Buxton, D. (2002): Chronic Meningoencephalitis Associated with Brucella sp. Infection in Live-stranded Striped Dolphins (Stenella coeruleoalba). J Comp Path, 126: 147-152.

Seidel, G.; Pardo, C. A.; Newman-Toker, D.; Olivi, A. and Eberhart, C. G. (2003): Neurobrucellosis Presenting 
as Leukoencephalopathy. The role of cytotoxic $\mathrm{T}$ lymphocytes. Arch Pathol Lab. Med., 127.

Guarda, F. (1977): Ulteriori ricerche Sulla neuropatologia dei feti bovini abortiti (Further research on the neuropathology of aborted fetuses). Annali Fac Med Vet Torino, 24: 1-28.

Hong, C. B.; Donahue, J. M.; Giles, R. C.; Poonacha, K. B.; Tuttle, P. A. and Cheville, N. F. (1991): Brucella abortus-associated meningitis in aborted bovine foetuses. Vet Pathol, 28: 492-496.

Hooper, P. T. (1999): Incidental lesions in the brain of sheep and goats. Aust Vet J, 77:398-399, [Web of Science][Medline]

Jeffrey, M. A. (1992): Neuropathological survey of brains submitted under the Bovine Spongiform Encephalopathy Orders in Scotland. Vet Rec 131:332-337.

Kikuchi, K. (1928): Über die altersveränderungen am gehirn des pferdes. Arch Tierheilkunde 58:541-573,

Labrisseau, A.; Marau, E. and Aguilera, F. (1978): The neurological complications of brucellosis. J Can Sci Neurol, 5: 369-376.

McLean, D.; Russell, N. and Khan, M. Y. (1992): Neurobrucellosis: clinical and therapeutic features. Clin Infect Dis, 15:582-590

Pappas, G.; Papadimitriou, P.; Akritidis, N.; Christou, L. and Tsianos, E. V. (2006): The new global map of human brucellosis. Lancet Infectious Diseases 6: 91-99.

Pascual, J.; Combarros, O.; Polo, J. M. and Berciano, J. (1988): Localized CNS brucellosis: report of 7 cases. Acta Neurol Scand, 78:282-9
Probert, W. S.; Schrader, K. N.; Khuong, N. Y. Bystrom, S. L. and Graves, M. H. (2004): Real-Time Multiplex PCR Assay for Detection of Brucella spp., B. abortus, and B. melitensis. J. Clin. Microbiol, 42(3): 12901293.

Sanchez-Sousa, A.; Torres, C.; Campello, M. G.; Garcia, C.; Parras, F.; Cercenado, E. And Baquero, F. (1990): Serological diagnosis of neurobrucellosis. Clin Pathol, 43:79-81.

Shaalan, M. A.; Memish, Z. A.; Mahmoud, S. A.; Alomari, A.; Khan, M. Y. and Almuneef M. and Alalola, S. (2002): Brucellosis in children: clinical observations in 115 cases. Int J Infect Dis, 6: 182-186.

Shakir, A. (1986): Neurobrucellosis. Postgrad Med J, 62: 1077-9.

Shakir, R. A.; Al-Din, A. S. N.; Araj. G. F.; Lulu, A. R.; Mousa, A. R. and Saadah, M. A. (1987): Clinical categories of neurobrucellosis, a report on 19 cases. Brain, 110:213-23.

Sözmen, M.; Erginsoy, S. D.; Genç O. .; Beytut, E. and Özcan, K. (2004): Immunohistochemical and Microbiological Detection of Brucella abortus in Aborted Bovine Fetuses. Acta Vet Brno, 73: 465-472.

Trujillo, I. Z.; Zavala, A. N.; Caceres, J. G. and Miranda, C. Q. (1994): Brucellosis infection. Infect Dis Clinic North Am, 8: 225-241.

Tuncel, D.; Uçmak, H.; Gokce, M. and Utku, U. (2008): Neurobrucellosis. Eur J Gen Med, 5(4):245-248.

Zaidan, R. and Al-Tahan, A. R. (1999): Cerebral venous thrombosis: a new manifestation of neurobrucellosis. Clin Infect Dis, 28:399-400. 